DEMONSTRATIO MATHEMATICA

Vol. XXXIV No $4 \quad 2001$

\author{
L. F. Barannyk, D. Klein
}

\title{
CROSSED GROUP RINGS WITH A FINITE SET OF DEGREES OF INDECOMPOSABLE REPRESENTATIONS OVER DEDEKIND DOMAINS
}

\begin{abstract}
Let $S$ be a Dedekind domain of characteristic $p>0, G$ be a finite group, $p|| G \mid$ and $S^{\lambda} G$ be a crossed group ring of the group $\mathrm{G}$ and of the ring $S$ with a factor system $\lambda$. Under some assumption on $G$ and $S$, we single out rings $S^{\lambda} G$ possessing finite set of degrees of indecomposable $S$-representations.
\end{abstract}

\section{Introduction}

Let $G$ be a finite group, $Z(G)$ the center of $G, G^{\prime}$ the commutant of $G$, $G_{p}$ a Sylow $p$-subgroup of $G, G_{p}^{\prime}$ a Sylow $p$-subgroup of $G^{\prime},|g|$ the order of $g \in G, \mathrm{~S}$ a commutative ring with an identity element, $S^{p}=\left\{a^{p}: a \in S\right\}$, $S^{*}$ the multiplicative group of the ring $S$, and $Z^{2}\left(G, S^{*}\right)$ the group of $S$ factor systems (2-cocycles) of the group $G$, where we assume that $G$ acts trivially on $S^{*}$ (see [23], Chapter 1). Any $S$-factor system of $G$ is equivalent to some normalized $S$-factor system of $G$. From now on we assume that $S$ factor systems of $G$ are normalized. A crossed group ring $S^{\lambda} G$ of the group $G$ and of the ring $S$ with a factor system $\lambda \in Z^{2}\left(G, S^{*}\right)$ is the $S$-algebra with $S$-basis $\left\{u_{g}: g \in G\right\}$ satisfying $u_{a} u_{b}=\lambda_{a, b} u_{a b}$ for all $a, b \in G$. The $S$-basis $\left\{u_{g}: g \in G\right\}$ of $S^{\lambda} G$ will be called natural. Let $e$ be the identity element of $G$. Then $u_{a} u_{e}=u_{e} u_{a}=u_{a}$ for all $a \in G$. We often identify $u_{e}$ with the identity element of the ring $S$. That is why, instead of $\gamma u_{e}$, we will write $\gamma(\gamma \in S)$. If $H$ is a subgroup of the group $G$, then the restriction of an $S$-factor system $\lambda \in Z^{2}\left(G, S^{*}\right)$ to $H \times H$ will also be denoted by $\lambda$. In this case $S^{\lambda} H$ is a subring of the ring $S^{\lambda} G$.

Let $\mathbb{M}_{n}(S)$ be the ring of all $n \times n$ matrices over $S$. A homomorphism of $S$-algebras $\Gamma: S^{\lambda} G \rightarrow \mathbb{M}_{n}(S)$ is called an matrix $S$-representation of $S^{\lambda} G$. The integer $n$ is the degree of $\Gamma$. Two matrix $S$-representations $\Gamma_{1}$ and $\Gamma_{2}$

1991 Mathematics Subject Classification: 16S35, 20C20, $20 \mathrm{C} 25$. 
of $S^{\lambda} G$ are said to be equivalent if there exist an invertible matrix $C$ over $S$ such that $C^{-1} \Gamma_{1}(v) C=\Gamma_{2}(v)$ for all $v \in S^{\lambda} G$. A matrix $S$-representation $\Gamma$ of the ring $S^{\lambda} G$ is said be decomposable if it is $S$-equivalent to a matrix $S$-representation $\Gamma^{\prime}$ of the form

$$
\Gamma^{\prime}(v)=\left(\begin{array}{cc}
\Gamma_{1}(v) & 0 \\
0 & \Gamma_{2}(v)
\end{array}\right) \quad\left(v \in S^{\lambda} G\right),
$$

where $\Gamma_{1}$ and $\Gamma_{2}$ are matrix $S$-representations of $S^{\lambda} G$. A matrix $S$-representation is indecomposable if it is not decomposable. By an $S^{\lambda} G$-module we mean a finitely generated left $S^{\lambda} G$-module which is $S$-free. The study of matrix $S$-representations of $S^{\lambda} G$ is essentially equivalent to the study of $S^{\lambda} G$-modules (see[7], §10).

Let $D\left(S^{\lambda} G\right)$ be the set of degrees of all indecomposable matrix $S$-representations of the crossed group ring $S^{\lambda} G$. If $S^{\lambda} G=S G$ is the usual group ring, that is $\lambda$ is trivial, then the problem whether $D\left(S^{\lambda} G\right)$ is finite, is solved in the following cases:

(a) $S$ is a field of characteristic $p[21]$;

(b) number ring [3-5], [9], [12], [19], [20], [22], [24], [27];

(c) ring of residue classes modulo $m$ [15];

(d) ring of polynomials in $t$ variables with coefficients from a field of characteristic $p$ [17];

(e) ring of formal power series in $t$ variables with coefficients from a complete discrete valuation ring [16];

(f) principal ideal domain of characteristic $p$ [13];

(g) local ring with residue class field of characteristic $p$ [18].

The case when $S^{\lambda} G$ is not equivalent to the group ring, that is $\lambda$ is not a coboundary, and $S$ is a ring of $P$-adic numbers or a field of characteristic $p$, is studied in [1], [2], [14] and [6].

We note that, in the case $S$ is a field, the set $D(S G)$ is finite if and only if the number of the equivalent classess indecomposable matrix $S$ representations of $S G$ is finite [21]. If $S$ is a principal ideal domain of characteristic 2 and is not a field, and if $G$ is the group of order 2 , then $D(S G)=$ $\{1,2\}[13]$ and there is an infinite number of pairwise non-equivalent indecomposable matrix $S$-representations of $S G$ [26].

For a complete discussion of related problems in the modern representation theory of finite groups, algebras, quivers and vector space categories the reader is referred to the monographs [10], [11], [23], [25], [28], [29].

In this paper we study the problem on finiteness of $D\left(S^{\lambda} G\right)$ under the assumption that $S$ is a Dedekind domain of characteristic $p>0$, and is not a field. 
Let us briefly present the main results of the paper. In Section 2, using the articles [13], [18] we prove that if $\left|G_{p}^{\prime}\right|>2$, then $D\left(S^{\lambda} G\right)$ is an infinite set for each cocycle $\lambda \in Z^{2}\left(G, S^{*}\right)$. The case where $G_{p}$ is abelian and $S=F[x]$ is the ring of polynomials in the variable $x$ over a field $F$ of characteristic $p$, is studied in Section 3. We establish that if $p \neq 2$ and $G_{p}$ is a normal subgroup of $G$, then $D\left(S^{\lambda} G\right)$ is a finite set if and only if $G=G_{p} \times H$ and $F^{\lambda} G_{p}$ is a field. Using a technique developed in [3], [4], we also prove a criterion of finiteness of $D\left(S^{\lambda} G\right)$ for $p=2$ and $G=G_{2}$. The criterion asserts that the set $D\left(S^{\lambda} G\right)$ is finite if and only if $\operatorname{dim}_{F}\left(F^{\lambda} G / \operatorname{rad} F^{\lambda} G\right) \geq \frac{|G|}{2}$, where $\operatorname{rad} F^{\lambda} G$ is the radical of the algebra $F^{\lambda} G$ (see [7],§24). In Section 4 , the analogous results are obtained for the case when $S=F[[x]]$ is a ring of formal power series in $x$ over the field $F$, and the cocycles are taken from $Z^{2}\left(G, F^{*}\right)$. Throughut this paper we use the terminology and notation introduced in [7], [8] and [23].

\section{Necessary conditions for finiteness of $D\left(S^{\lambda} G\right)$}

Let $K$ be a quotient field of $S, P$ be a maximal ideal of $S, \pi \in P$ and $\pi \notin P^{2}, \varphi$ be the $P$-adic valuation of $K, \hat{K}$ be the $\varphi$-adic completion of $K$, $\hat{S}$ be the valuation ring of $\hat{K}$ and $\hat{P}$ be the maximal ideal of $\hat{S}$. As we know $([8], \S 4 \mathrm{C}), \hat{P}=\pi \hat{S}$ and $S / P \cong \hat{S} / \hat{P}$.

Let $\lambda \in Z^{2}\left(G, S^{*}\right)$. If $a \in G$ is an element of order $n$, then the equality

$$
u_{a}^{n}=\lambda_{a, a} \lambda_{a, a^{2}} \ldots \lambda_{a, a^{n-1}} u_{e}
$$

holds in $S^{\lambda} G$.

Let $G=\left(a_{1}\right) \times \ldots \times\left(a_{m}\right)$ be an abelian group of type $\left(p^{r_{1}}, \ldots, p^{r_{m}}\right)$. For each cocycle $\lambda \in Z^{2}\left(G, S^{*}\right)$, the ring $S^{\lambda} G$ is commutative. A natural $S$-basis $\left\{u_{g}: g \in G\right\}$ of the ring $S^{\lambda} G$ can be chosen in such a way that the following conditions are satisfied:

(1) if $a=a_{1}^{i_{1}} \ldots a_{m}^{i_{m}} \in G$, where $0 \leq i_{q}<p^{r_{q}}(q=1, \ldots, m)$, then $u_{a}=u_{a_{1}}^{i_{1}} \ldots u_{a_{m}}^{i_{m}}$,

(2) $u_{a_{j}}^{p^{r_{j}}}=\delta_{j}\left(\delta_{j} \in S^{*} ; j=1, \ldots, m\right)$.

In this connection, we also denote the ring $S^{\lambda} G$ by $\left[G, S, \delta_{1}, \ldots, \delta_{m}\right]$.

2.1. Lemma. Let $S$ be a Dedekind domain of characteristic $p$. Assume that either $p \neq 2$ and $G$ contains an element $a$ of order $p$ such that

$$
\lambda_{a, a} \lambda_{a, a^{2}} \ldots \lambda_{a, a^{p-1}}=\mu^{p}, \quad \text { for some } \mu \in S^{*},
$$

or $p=2$ and $G$ contains an element $a$ of order 4 such that

$$
\lambda_{a, a} \lambda_{a, a^{2}} \lambda_{a, a^{3}}=\mu^{4}, \quad \text { for some } \mu \in S^{*} .
$$

Then $D\left(S^{\lambda} G\right)$ is an infinite set. 
Proof. Let

$$
\hat{S}^{\lambda} G=\hat{S} \otimes_{S} S^{\lambda} G,
$$

$H=(a), E_{n}$ be the identity matrix of order $n, J_{n}$ be the Jordan block of order $n$ with the unity on the main diagonal. By virtue of Lemma 2 in [13], the $\hat{S}$-linear mapping $\hat{\Gamma}: \hat{S}^{\lambda} H \rightarrow \mathbb{M}_{3 n}(\hat{S})$ defined by

$$
\hat{\Gamma}\left(u_{a}\right)=\mu\left(\begin{array}{ccc}
E_{n} & \pi E_{n} & J_{n} \\
0 & E_{n} & \pi E_{n} \\
0 & 0 & E_{n}
\end{array}\right),
$$

is an indecomposable $\hat{S}$-representation of the ring $\hat{S}^{\lambda} H$. Since for $\hat{S}$-representations of the ring $\hat{S}^{\lambda} G$, the Krull-Schmidt Theorem [5] is true, we conclude that in virtue of [14], one of the indecomposable summands of the induced representation

$$
\hat{\Gamma}^{\hat{S}^{\lambda} G}
$$

of the ring $\hat{S}^{\lambda} G$ has degree greater than or equal to $3 n$.

Let $\Gamma$ be the restriction of $\hat{\Gamma}$ to $S^{\lambda} H$. Then $\Gamma$ is an $S$-representation of the ring $S^{\lambda} H$ and the representation (2.1) can be obtained from the $S$-representation

$$
\Gamma^{S^{\lambda} G}
$$

of the ring $S^{\lambda} G$ as a result of extension of the ring of scalars $S$ up to the ring $\hat{S}$. Therefore, a decomposition of the representation (2.2) in a direct sum of indecomposable representations has a summand of degree greater than or equal to $3 n$. The lemma is proved.

2.2. Lemma. Let $S$ be a Dedekind domain of characteristic 2 . Assume that $G$ contains a subgroup $H=(a) \times(b)$ of type $(2,2)$ such that $\lambda_{a, a}=\gamma^{2}, \lambda_{b, b}=\delta^{2}$ for some $\gamma, \delta \in S^{*}$. Then $D\left(S^{\lambda} G\right)$ is an infinite set.

Proof. By Lemma 4 [18], the $\hat{S}$-representation $\hat{\Gamma}$ of the ring $\hat{S}^{\lambda} H$, defined by the equalities

$$
\hat{\Gamma}\left(u_{a}\right)=\gamma\left(\begin{array}{cc}
E_{n} & E_{n} \\
0 & E_{n}
\end{array}\right), \quad \hat{\Gamma}\left(u_{b}\right)=\delta\left(\begin{array}{cc}
E_{n} & J_{n} \\
0 & E_{n}
\end{array}\right)
$$

is indecomposable. Then we use the same reasonings as in the proof of Lemma 2.1.

2.3. Lemma. Let $S$ be a Dedekind domain of characteristic $p, H$ be a $p$ subgroup of $G^{\prime}$ and $\lambda_{H}: H \times H \rightarrow S^{*}$ be the restriction of a cocycle $\lambda \in$ $Z^{2}\left(G, S^{*}\right)$ to $H \times H$. Then $\lambda_{H}$ is a coboundary. 
P r o of. Since $S^{*}$ has no elements of order $p$, the Lemma 2.3 follows by virtue of Corollary 4.10 [23, p. 42].

2.4. THEOREM. Let $S$ be a Dedekind domain of characteristic $p \neq 2$. If the set $D\left(S^{\lambda} G\right)$ is finite for some $\lambda \in Z^{2}\left(G, S^{*}\right)$, then $G=H \rtimes G_{p}$ and $G_{p}$ is abelian. Moreover, if $F$ is a subfield of $S$ and $\lambda \in Z^{2}\left(G, F^{*}\right)$, then $F^{\lambda} G_{p}$ is a field.

Proof. By virtue of Lemmas 2.1 and $2.3, p$ does not divide $\left|G^{\prime}\right|$. It then follows that $H=\{g \in G:|g| \not \equiv 0(\bmod p)\}$ is a normal subgroup of $G$. By Schur's Theorem, $G=H \rtimes G_{p}$. Let $G_{p}=\left(a_{1}\right) \times \ldots \times\left(a_{m}\right)$ be a group of type $\left(p^{r_{1}}, \ldots, p^{r_{m}}\right), S^{\lambda} G_{p}=\left[G_{p}, S, \delta_{1}, \ldots, \delta_{m}\right]$, where $\delta_{j} \in F^{*}(j=1, \ldots, m)$. Assume that $F\left[u_{a_{1}}, \ldots, u_{a_{j}}\right]$ is a field and $F\left[u_{a_{1}}, \ldots, u_{a_{j}}, u_{a_{j+1}}\right]$ is not a field. It follows that $\delta_{j+1} u_{e}=v^{p}$, where $v \in F\left[u_{a_{1}}, \ldots, u_{a_{j}}\right]$. Let

$$
\begin{aligned}
& b_{i}=a_{i}^{p^{r_{i}-1}}, \quad u_{b_{i}}=u_{a_{i}}^{p^{r_{i}-1}} \quad(i=1, \ldots, m), \\
& w=\delta_{j+1}^{-1} v^{p-1} u_{b_{j+1}}, \quad B=\left(b_{1}\right) \times \ldots \times\left(b_{m}\right) .
\end{aligned}
$$

We have

$$
\begin{gathered}
u_{b_{i}}^{p}=\delta_{i} u_{e}, \quad w^{p}=u_{e}, \quad u_{b_{j+1}}=v w \\
v \in F\left[u_{b_{1}}, \ldots, u_{b_{j}}\right], \quad F\left[u_{b_{1}}, \ldots, u_{b_{j}}, u_{b_{j+1}}\right]=F\left[u_{b_{1}}, \ldots, u_{b_{j}}, w\right] .
\end{gathered}
$$

Therefore, $S^{\lambda} B=S^{\mu} N=\left[N, S, \delta_{1}, \ldots, \delta_{j}, 1, \delta_{j+2}, \ldots, \delta_{m}\right]$, where $N$ is an elementary abelian $p$-group of order $|B|$. Let

$$
\Gamma(w)=\left(\begin{array}{ccc}
E_{n} & \pi E_{n} & J_{n} \\
0 & E_{n} & \pi E_{n} \\
0 & 0 & E_{n}
\end{array}\right) .
$$

Arguing as in the proof of Lemma 2.1, we obtain that any of the indecomposable components of the induced representation

$$
\left(\Gamma^{S^{\mu} N}\right)^{S^{\lambda} G}
$$

of the ring $S^{\lambda} G$ has degree greater than or equal to $3 n$. This contradicts the assumption that $D\left(S^{\lambda} G\right)$ is a finite set. Therefore, $F^{\lambda} G_{p}$ is a field, and the proof is completed.

2.5. TheOREM. Let $S$ be a Dedekind domain of characteristic 2 . If the set $D\left(S^{\lambda} G\right)$ is finite for some $\lambda \in Z^{2}\left(G, S^{*}\right)$, then 4 does not divide $\left|G^{\prime}\right|$. Moreover, if $G=G_{2} \rtimes H$, then $G=G_{2} \times H$.

Proof. The first statement follows immediately from Lemmas $2.1-2.3$. Let $G=G_{2} \rtimes H$ and $\left[G_{2}, h\right] \neq\{e\}$ for some $h \in H$. Assume that $G_{2}$ is abelian. If $a, b$ are non-identity elements of $G_{2}$ and $h^{-1} a h=a b$, then $b^{2}=e$ and $h^{-1} b h=b$. In this case, $h^{-2} a h^{2}=a$. But $(h)=\left(h^{2}\right)$, hence, 
$h^{-1} a h=a$. The contradiction obtained proves that $G_{2}$ is a non-abelian group. Then $G_{2}^{\prime}=(c)$, where $c \in Z(G)$. Let $\bar{G}=\left(G_{2}, h\right)$. Using formula $[x y, z]=\left[x^{y}, z^{y}\right] \cdot[y, z]$ we get $[\bar{G}, \bar{G}]=\left[\bar{G}, G_{2}\right]=(c)$. In this case, $\bar{G}$ is a nilpotent group. This contradicts to $\left[G_{2}, h\right] \neq\{e\}$, and the proof is finished.

\section{Crossed group rings with a finite set of degrees of indecompo- sable representations over a ring of polynomials}

In this section, we assume that $S=F[x]$ is a ring of polynomials in the variable $x$ over a field $F$ of characteristic $p$. As known, if $P=x S$ (we use the notation of Sec. 2), then $\hat{S}=F[[x]]$ is the ring of formal power series in the variable $x$ with coefficients from the field $F$. Since $S^{*}=F^{*}$, sometimes we write $Z^{2}\left(G, F^{*}\right)$ instead of $Z^{2}\left(G, S^{*}\right)$. Let us note that in the case under consideration $K=F(x)$.

3.1. THEOREM. Let $F$ be a field of characteristic $p \neq 2, S=F[x], G=G_{p} \rtimes$ $H$, and $\lambda \in Z^{2}\left(G, S^{*}\right)$. The set $D\left(S^{\lambda} G\right)$ is finite if and only if $G=G_{p} \times H$, $G_{p}$ is an abelian group and $F^{\lambda} G_{p}$ is a field.

Proof. The necessity of the theorem follows from Theorem 2.4. Let us prove the sufficiency. The ring $S^{\lambda} G_{p}$ can be viewed as the ring of polynomials in the variable $x$ over the field $L=F^{\lambda} G_{p}$. Because of this, $S^{\lambda} G$ is a crossed group ring $R^{\nu} H$ of the group $H$ and the ring $R=L[x]$. Let $M$ be a finitely generated $S$-torsion free $S^{\lambda} G$-module. Then $M$ is a finitely generated $R^{\nu} H$ module. If $m \in M, m \neq 0$ and $f(x) m=0$ for some $f(x) \in L[x]$, then

$$
(f(x))^{p^{n}} m=0
$$

where $p^{n}=\exp G_{p}$. But

$$
(f(x))^{p^{n}} \in F[x]=S,
$$

therefore, $(f(x))^{p^{n}}=0$, whence, $f(x)=0$. This proves that $M$ is an $R$ torsion free module. The isomorphism and the indecomposability of $S^{\lambda} G$ modules are equivalent to those of $R^{\nu} H$-modules. Since $p \nmid|H|$, each of the $R^{\nu} H$-modules is a completely reducible module. It is known [7] that $R$-rank of each of the irreducible $R^{\nu} H$-modules does not exceed $|H|$. Therefore, $D\left(S^{\lambda} G\right)$ is a finite set, and the proof is finished.

Let $t$ be a root of a polynomial of degree $m+1$ with the leading coefficient 1 which is irreducible over $F$. Let us denote by $\tilde{t}$ the matrix correspoding to the operator of the multiplication by $t$ in the $F$-basis $1, t, \ldots, t^{m}$ of the field $F[t]$.

3.2. Lemma. Let $F$ be a field of characteristic $2, G=(a)$ a cyclic group of order $2^{n}(n \geq 1), S=F[x]$, and $S^{\lambda} G=\left[G, S, \alpha^{2^{m}}\right]$, where $\alpha \notin F^{2}$. If $m=0$, 
then up to the equivalence, the ring $S^{\lambda} G$ has only one indecomposable $S$ representation $u_{a} \rightarrow \tilde{\theta}$, where $\theta$ is a root of the irreducible polynomial

$$
y^{2^{n}}-\alpha \in F[y] \text {. }
$$

If $m=1$, then up to equivalence, the indecomposable $S$-representations of the ring $S^{\lambda} G$ are the following:

$$
\tilde{\rho}, \quad\left(\begin{array}{cc}
\tilde{\rho} & \langle f(x, \rho)\rangle \\
0 & \tilde{\rho}
\end{array}\right),
$$

where $\rho$ is a root of the irreducible polynomial

$$
y^{2^{n-1}}-\alpha \in F[y]
$$

and $\langle f(x, \rho)\rangle$ is the matrix in which all columns but last one being zero, and the last column consisting of the coordinates of $f(x, \rho) \in S[\rho]$ in the $S$-basis

$$
1, \rho, \ldots, \rho^{2^{n-1}-1}
$$

of the ring $S[\rho]$.

Proof. If $m=0$, then $S^{\lambda} G \cong S[\theta]=F[\theta][x]$, where $F[\theta]$ is a field. Each $S^{\lambda} G$-module can be considered as a torsion-free module over the principal ideal ring $S[\theta]$, therefore, $M \cong S[\theta] \oplus \ldots \oplus S[\theta]$. Hence, up to equivalence, the ring $S^{\lambda} G$ has only one indecomposable $S$-representation $u_{a} \rightarrow \tilde{\theta}$. Let $m=1, M$ be an arbitrary $S^{\lambda} G$-module, and

$$
N=\left\{v \in M:\left(u_{a}^{2^{n-1}}-\alpha\right) v=0\right\} .
$$

One can view the $S^{\lambda} G$-module $N$ as a module over the ring $S^{\lambda} G /\left(u_{a}^{2^{n-1}}-\alpha\right) S^{\lambda} G \cong S[\rho]$. Since $S[\rho]$ is a principal ideal ring and $N$ is a $S[\rho]$-torsion free module, we get

$$
N \cong S[\rho] \oplus \ldots \oplus S[\rho] .
$$

The $S^{\lambda} G$-module $M / N$ can also be viewed as a $S[\rho]$-module. Since $\mathrm{M}$ is a free $S$-module, $N$ is an $S$-pure submodule of the module $M$ (see [8], p.84). If $\varphi(x, \rho) \in S[\rho]$, then

$$
\varphi(x, \rho)^{2^{n-1}} \in S
$$

and, therefore, the equality $\varphi(x, \rho)[v+N]=N$ yields $\varphi(x, \rho)=0$ or $v \in N$. This means that $M / N$ is a torsion-free module over $S[\rho]$. Therefore,

$$
M / N \cong S[\rho] \oplus \ldots \oplus S[\rho] .
$$

Using (3.1), (3.2) and the $S$-purity of the module $N$, we conclude that any $S$-representation $\Gamma$ of the ring $S^{\lambda} G$ which is realized in the $S^{\lambda} G$-module $M$ can be written in the form

$$
\Gamma\left(u_{a}\right)=\left(\begin{array}{cc}
\tilde{\rho}^{(r)} & * \\
0 & \tilde{\rho}^{(t)}
\end{array}\right)
$$


where $\tilde{\rho}^{(t)}=\operatorname{diag}\left[\tilde{\rho}_{1}, \ldots, \tilde{\rho}_{t}\right]$ and $\rho_{1}=\ldots=\rho_{t}=\rho$. Using the technique developed in [3], [4], we conclude that indecomposable $S$-representations of the ring $S^{\lambda} G$ for $m=1$ are the following representations:

$$
\tilde{\rho}, \quad\left(\begin{array}{cc}
\tilde{\rho} & \langle f(x, \rho)\rangle \\
0 & \tilde{\rho}
\end{array}\right) .
$$

The lemma is proved.

3.3. THEOREM. Let $F$ be a field of characteristic $2, S=F[x]$, and $G$ be an abelian 2-group. The set $D\left(S^{\lambda} G\right)$ is finite if and only if $\operatorname{dim}\left(F^{\lambda} G / \operatorname{rad} F^{\lambda} G\right)$ $\geq \frac{|G|}{2}$.

Pr o of. If $D\left(S^{\lambda} G\right)$ is finite, then using Lemma 2.2 and reasonings presented in the proof of Theorem 2.4, we conclude that there exists a decomposition $G=H \times(b)$ such that $T=F^{\lambda} H$ is a field. Let $L=T[x]$, and $B=(b)$. The ring $S^{\lambda} G$ can be viewed as a crossed group ring

$$
L^{\mu} B=\left[B, L, \gamma^{2^{m}}\right]
$$

where either $\gamma=1, m=0$ or $\gamma \in T, \gamma \notin T^{2}$ and $2^{m}<|b|$. Since $D\left(S^{\lambda} G\right)$ is finite if and only if $D\left(L^{\mu} B\right)$ is finite, then by Lemma 2.1 , we get $m \leq 1$. This means

$$
\operatorname{dim}\left(F^{\lambda} G / r a d F^{\lambda} G\right) \geq \frac{|G|}{2}
$$

The converse statement can be proved by the same reasonings and using Lemma 3.2.

3.4. Corollary. Let $F$ be a field of characteristic $2, S=F[x], G$ be an abelian 2-group, and $H=\left\{g \in G: g^{4}=e\right\}$. The set $D\left(S^{\lambda} G\right)$ is finite if and only if the set $D\left(S^{\lambda} H\right)$ is finite.

3.5. REMARK. Let $F$ be a field of characteristic $2, S=F[x], G$ be an abelian 2-group, and $N$ be the socle of $G$. If $D\left(S^{\lambda} G\right)$ is a finite set, then the set $D\left(S^{\lambda} N\right)$ is also finite. The converse does not hold.

\section{Crossed group rings with a finite set of degrees of indecompos- able representations over a ring of formal power series}

We assume that $F$ is a field of characteristic $p>0, S=F[[x]], G$ is a finite group, $G_{p}$ is a Sylow $p$-subgroup of the group $G$. If $\Omega$ is an algebraic closure of the field $F$, then we assume $F[[x]] \subset \Omega[[x]]$. Therefore, for each $\theta \in \Omega$ we have $F[[x]][\theta]=F[\theta][[x]]$. Theorem 3.3 is also valid for rings $F[[x]]^{\lambda} G$ under the same assumption $\lambda \in Z^{2}\left(G, F^{*}\right)$.

4.1. Proposition. Let $F$ be a field of characteristic $p \neq 2, S=F[[x]]$, $G=H \rtimes G_{p}$ and $G_{p}$ be an abelian group. If $\lambda \in Z^{2}\left(G, F^{*}\right)$, then $D\left(S^{\lambda} G\right)$ is finite if and only if $F^{\lambda} G_{p}$ is a field. 
Proof. Apply Theorem 2.4 and Higman's theory of relative projectivity [21] that was developed in $[1,14]$ for crossed group rings.

We remark that if $G=H \rtimes G_{p}$, then for each $\mu \in Z^{2}\left(G_{p}, F^{*}\right)$ there exists a $\lambda \in Z^{2}\left(G, F^{*}\right)$ such that $\lambda_{G_{p} \times G_{p}}=\mu$.

4.2. Proposition. Let $F$ be a field of characteristic $2, S=F[[x]], G$ be a finite group, $\left|G_{2}^{\prime}\right| \neq 2$, and $\lambda \in Z^{2}\left(G, F^{*}\right)$. The set $D\left(S^{\lambda} G\right)$ is finite if and only if $G=H \rtimes G_{2}, G_{2}$ is abelian and $\operatorname{dim}\left(F^{\lambda} G_{2} / r a d F^{\lambda} G_{2}\right) \geq \frac{\left|G_{2}\right|}{2}$.

Proof. Apply Theorem 2.5 and an analogue of Theorem 3.3 for $F[[x]]$.

4.3. Proposition. Let $F$ be a perfect field of characteristic $p, S=F[[x]]$, $G$ a finite group, and $\lambda \in Z^{2}\left(G, F^{*}\right)$. The set $D\left(S^{\lambda} G\right)$ is finite if and only if $p \nmid|G|$ for $p \neq 2$ and $4 \nmid|G|$ for $p=2$.

Proof. Since $S^{\lambda} G_{p}$ is equivalent to the group ring $S G_{p}[23$, p.68], the Proposition 4.3 follows by virtue of Theorem 1 [13].

4.4. Proposition. Let $F$ be a perfect field of characteristic $p \neq 2, S=$ $F[[x]], G$ a finite group, $\lambda \in Z^{2}\left(G, S^{*}\right)$. Suppose that the group $G$ contains an element $a$ of order $p$ such that

$$
\lambda_{a, a} \lambda_{a, a^{2}} \ldots \lambda_{a, a^{p-1}}=\gamma+x \sigma,
$$

where $\gamma \in F, \sigma \in S$ and $\sigma \not \equiv 0(\bmod x)$. Then the set $D\left(S^{\lambda} G\right)$ is finite if and only if $G_{p}$ is a cyclic group.

$\operatorname{Pr}$ oof. Since $F$ is a perfect field, then going (if necessary) to the equivalent factor set, we can assume $\lambda_{g_{1}, g_{2}} \equiv 1(\bmod x)$ for arbitrary $g_{1}, g_{2} \in G_{p}$. In particular, in (4.1) we have $\gamma=1$.

Assume that $D\left(S^{\lambda} G\right)$ is a finite set. Then by virtue of Theorem 2.4, $G_{p}$ is an abelian group. Let $H$ be the socle of $G_{p}$ and $S^{\lambda} H=\left[H, S, \delta_{1}, \ldots, \delta_{m}\right]$. Without loss of generality, we can assume $\delta_{1} \equiv 1(\bmod x)$ and $\delta_{1} \not \equiv 1\left(\bmod x^{2}\right)$. Let $\tilde{x}=\delta_{1}-1$. Then $x S=\bar{x} S$. Hence, each element $\mu \in S$ can be presented as a formal series $\mu=\gamma_{0}+\gamma_{1} \bar{x}+\gamma_{2} \bar{x}^{2}+\ldots$, where $\gamma_{j} \in F(j=0,1,2, \ldots)$. Let $\theta$ be a root of the polynomial $y^{p}-\delta_{1}$ and $\pi=\theta-1$. Then $\pi^{p}=\bar{x}$, and hence

$$
\mu=\left(\rho_{0}+\rho_{1} \pi+\rho_{2} \pi^{2}+\ldots\right)^{p},
$$

where $\rho_{j}^{p}=\gamma_{j}$ and $\rho_{j} \in F$ for each $j=0,1,2, \ldots$ It follows that $\delta_{i}=\omega_{i}^{p}$, where $\omega_{i} \in S[\theta]$ for each $i=2, \ldots, m$. By the same reasonings as in the proof of Theorem 2.4 we obtain that if $m \neq 1$, then $D\left(S^{\lambda} G\right)$ is an infinite set. Therefore, $G_{p}$ is cyclic.

Conversely, assume that $G_{p}=(b)$, where $|b|=p^{n}$. On the basis of (4.1) we get

$$
u_{b}^{p^{n}}=1+\bar{x}
$$


where $\bar{x} \in S$ and $\bar{x} S=x S$. It follows that $S\left[u_{b}\right]$ is the ring of all $S$-integral elements of the field $K\left[u_{b}\right]$, where $K$ is the quotient field of the ring $S$. Since $S\left[u_{b}\right]$ is a principal ideal ring, each finitely generated $S$-torsion free $S\left[u_{b}\right]-$ module is isomorphic to $S\left[u_{b}\right] \oplus \ldots \oplus S\left[u_{b}\right]$. Then the finiteness of $D\left(S^{\lambda} G\right)$ follows from the finiteness of $D\left(S^{\lambda} G_{p}\right)$. The proposition is proved.

4.5. Proposition. Let $F$ be a perfect field of characteristic $2, S=F[[x]]$, $G$ a finite group, $G_{2}$ a Sylow 2-subgroup of $G$, and $\lambda \in Z^{2}\left(G, S^{*}\right)$. Suppose that $G_{2}$ is abelian and $G_{2}$ contains an element $a$ of order 4 such that $\lambda_{a, a} \lambda_{a, a^{2}} \lambda_{a, a^{3}}=\gamma+x \rho$, where $\gamma \in F, \rho \in S$ and $\rho \neq \equiv(\bmod x)$. Then $D\left(S^{\lambda} G\right)$ is a finite set if and only if $G_{2}$ is a cyclic group or group of type $\left(2^{n}, 2\right)$.

Proof. We can assume $G_{2}=\left(a_{1}\right) \times \ldots \times\left(a_{m}\right),\left|a_{i}\right|=2^{n_{i}}(i=1, \ldots, m)$, $n_{1} \geq 2$ and

$$
u_{a_{1}}^{2^{n_{1}}}=1+x .
$$

The ring $S^{\lambda} G_{2}$ can be viewed as a crossed group ring of the group $\left(a_{2}\right) \times$ $\ldots \times\left(a_{m}\right)$ and of the ring $S\left[u_{a_{1}}\right]$. Let $\theta$ be a root of the polynomial

$$
y^{2^{n_{1}}}-x \text {. }
$$

Then $S\left[u_{a_{1}}\right] \cong S[\theta]$ and each element $\mu \in S$ can be expressed as $\mu=\rho^{2^{n_{1}}}$, where $\rho \in S[\theta]$. Hence, by Lemmas 2.1 and 2.2, we conclude that if $D\left(S^{\lambda} G\right)$ is a finite set, then $m \leq 2$, and, if $m=2$, then $n_{2}=1$. This proves the necessity.

Let us prove the sufficiency. If $G_{2}$ is cyclic, then $S^{\lambda} G_{2} \cong S[\theta]$. Then we use the same reasonings as in the proof of Proposition 4.4. If $G_{2}$ is of type $\left(2^{n}, 2\right)$, then $S^{\lambda} G_{2}$ can be viewed as a group ring of the group of order 2 and of the ring $S[\theta]$. The set of degrees of indecomposable $S(\theta)$-representations of such a group ring is $\{1,2\}$ [13]. Therefore, $D\left(S^{\lambda} G_{2}\right)=\left\{2^{n}, 2^{n+1}\right\}$. The finiteness of $D\left(S^{\lambda} G\right)$ follows from the finiteness of $D\left(S^{\lambda} G_{2}\right)$. The proposition is proved.

Acknowledgment. We are grateful to the referee for valuable remarks.

\section{References}

[1] L. F. Barannyk and P. M. Gudivok, Projective representations of finite groups over number rings, Mat. Sbornik, 82 (124) (1970), 423-443=Math. USSR Sb., 11 (1970), 391-410.

[2] L. F. Barannyk and P. M. Gudivok, Crossed group rings of finite groups and rings of $P$-adic integers with finitely many indecomposable integral representations, Mat. Sbornik. 108(150)(1979), 187-211=Math. USSR., 36(1980), 173-194. 
[3] S. D. Berman and P. M. Gudivok, Integral representations of finite groups, Dokl. Akad. Nauk SSSR, 145 (1962), 1199-1201=Soviet Math. Dokl., 3 (1962), 1172-1174.

[4] S. D. Berman and P. M. Gudivok, Indecomposable representations of finite groups over the ring of $p$-adic integers, Izv. Akad. Nauk SSSR, Ser. Mat. 28 (1964), 875910, English transl., Amer. Math. Soc. Transl., (2) 50 (1966), 77-113.

[5] Z. I. Borevich and D. K. Faddeev, Theory of homology in groups, II. Vestnik Leningrad. Univ. 14 (1959), No 7, 72-87 (in Russian).

[6] S. B. Conlon, Twisted group algebras and their representations, J. Austral. Math. Soc. 4 (1964), 152-173.

[7] C. W. Curtis and I. Reiner, Representation Theory of Finite Groups and Associative Algebras, New York: Interscience, 1962; 2nd ed., 1966.

[8] C. W. Curtis and I. Reiner, Methods of Representation Theory, Vol. I, New York: Wiley, 1981.

[9] F. E. Diederichsen, Über die Ausreduktion ganzzahliger Gruppendarstellungen bei arithmetischer Aquivalenz, Abh., Math. Sem. Univ. Hamburg, 14 (1940), 357-412.

[10] J. A. Drozd and V. V. Kirichenko, Finite Dimensional Algebras, Springer, Berlin, 1994.

[11] P. Gabriel and A. V. Roiter, Representations of Finite Dimensional Algebras. Algebra VIII, Encyclopedia of Math. Sc., Vol. 73, Springer, 1992; 2 nd print., Springer, Berlin, 1997.

[12] P. M. Gudivok, Representations of finite groups over number rings, Izv. Akad. Nauk SSSR, Ser. Mat., 31 (1967), 799-834=Math. USSR Izv., 1(1967), 773-805.

[13] P. M. Gudivok, On boundedness of degrees of indecomposable modular representations of finite groups over principal ideal rings, Dopovidi Akad. Nauk USSR, Ser. A(1971), No 8, 683-685 (in Ukrainian).

[14] P. M. Gudivok, On the number of indecomposable integral p-adic representations of crossed group rings, Mat. Sb., 91(133) (1973), 27-49=Math. USSR Sb., 20 (1973).

[15] P. M. Gudivok, V. S. Drobotenko and A. I. Lichtman, On representations of finite groups over the ring of residue classes mod $m$, Ukrain. Math. J., 16 (1964), 82-89 (in Russian).

[16] P. M. Gudivok, V. M. Oros and A. V. Roiter, On representations of finite $p$ groups over a ring of formal power series with p-adic integer coefficients, Ukrain. Math. J. 44 (1992), No 6, 753-765 (in Russian).

[17] P. M. Gudivok and E. Ya. Pogorilyak, On modular representations of finite groups over integral domains, Proc. Steklov Inst. Math., No 4 (1991), 87-95.

[18] P. M. Gudivok and V. I. Pogorily ak, On indecomposable representations of finite p-groups over commutative local rings, Dopovidi Nat. Akad. Nauk Ukraïny (1996), No 5, 7-11 (in Russian).

[19] A. Heller and I. Reiner, Representations of cyclic groups rings of integers I, Ann. Math., 76 (1962), 73-92.

[20] A. Heller and I. Reiner, Representations of cyclic groups rings of integers $I I$, Ann. Math. 77 (1963), 318-328.

[21] D. G. Higman, Indecomposable representations at characteristic $p$, Duke Math. J. 21 (1954), 377-381.

[22] H. Jacobinski, Sur les ordres commutatifs avec un nombre fini de reseaux indecomposables, Acta Math. 118 (1967), 1-31.

[23] G. Karpilovsky, Group representations, North-Holland Mathematical Studies 177, 1993. 
[24] L. A. Nazarova, Unimodular representations of the four group, Dokl. Akad. Nauk SSSR, 140 (1961), 1011-1014=Soviet Math. Dokl., 2 (1961), 1304-1307.

[25] J. Okniński, Semigroups of Matrices, Series in Algebra, Vol. 6, World Scientific, London, 1998.

[26] K. W. Roggenkamp, Gruppenringe von unendlichem Darstellungstyp, Mat. Z. 96 (1967), 393-398.

[27] A. V. Roiter, On representations of the cyclic group of fourth order by integral matrices, Vestnik Leningrad. Univ, 15 (1960), No 19, 65-74 (in Russian).

[28] A. H. Schofield, Representations of Rings over Skew Fields, London Math. Soc. Lecture Notes Series, No. 92 (Cambridge University Press, 1985).

[29] D. Simson, Linear Representations of Partially Ordered Sets and Vector Space Categories, Algebra, Logic and Applications, Vol. 4, Gordon \& Breach Science Publishers, Switzerland-Australia, 1992.

\section{INSTITUTE OF MATHEMATICS \\ PEDAGOGICAL ACADEMY \\ Arciszewskiego 22b \\ 76-200 SEUPSK, POLAND \\ E-mail: barannyk@gryf2.wsp.slupsk.pl \\ klein@gryf2.wsp.slupsk.pl}

Received June 29, 2000; revised version January 18, 2001. 